\title{
THE YIN AND YANG OF A DOCTOR OF SCIENCE IN INFORMATION SYSTEMS AND COMMUNICATIONS PROGRAM: PERSONAL REFLECTIONS AND REVERSE ENGINEERING
}

\author{
Fred Hoffman, Mercyhurst University, fhoffman@mercyhurst.edu \\ Chaojie Wang, The MITRE Corporation, cjwang@mitre.org* \\ Alvi Lim, The MITRE Corporation, alim@mitre.org* \\ Jin Kwon, Robert Morris University, jhkst187@mail.rmu.edu
}

\begin{abstract}
This reflective paper provides fresh accounts of the four co-authors' personal experiences with respect to successfully completing the Doctor of Science in Information Systems and Communications program at Robert Morris University. This paper also presents a Yin \& Yang Research Model that integrates the ancient Chinese concept of Yin and Yang and the modern Western concept of the Data-Information-Knowledge-Wisdom hierarchy. This model reflects the complementary nature of qualitative and quantitative research methodologies. The unusually high graduation rate of this program over two decades, coupled with the subsequent, successful career trajectories of over 200 graduates, attest to the effective balance of humanity and technology that characterizes this program. The mix of doctoral courses offered within this program, coupled with the technical expertise and philosophical and research orientations of the associated faculty members, is fully consistent with this Yin and Yang concept of balance and harmony. The program was able to accommodate and individually benefit the four authors, each of whom came from a different national cultural heritage and birth country, had a different professional and technical background, and employed a different research methodology from the other three in their doctoral dissertation. This paper also illustrates the benefits of a cohort-based, resident, executive format doctoral program that emphasizes support and collaboration, knowledge sharing and co-creation. While four recent graduates represent an admittedly small and biased sample of the program's 200+ graduates, the authors nevertheless believe their reflections, considered within the framework of the Yin \& Yang Research Model, provide valuable insights useful for comprehending some of the reasons for the program's consistently high graduation rate. Such insights may be useful to other institutions seeking to establish a new, similar program, or to improve an existing one. In addition, this paper provides practical and theoretical perspectives to help prospective students considering the pursuit of a terminal degree, in the process of program selection, or already on their selected path of higher learning.
\end{abstract}

Keywords: Doctoral Program, Information Systems, Humanity, Social Science, Higher Education

\section{INTRODUCTION}

2019 marks the $20^{\text {th }}$ anniversary of the Doctor of Science in Information Systems and Communications (DISC) program at Robert Morris University (RMU). The DISC program has proven to be highly successful over the two decades of its existence, consistently sustaining a more than $90 \%$ graduation rate, a rate that is nearly double that of other doctoral programs (Mishra, Cellante, \& Kohun, 2018). Success of the DISC program extends well beyond its graduation rate: Its more than 200 graduates since 2002 have generated over a thousand peer-reviewed publications, and while some DISC program graduates went on to become professors and researchers and thrive in academic and scientific pursuits, others have gone on to attain senior executive leadership positions in government and in the commercial sector.

Why has this program been so successful, and how has it avoided the roughly $50 \%$ attrition rate of other doctoral programs? Several papers have addressed these questions, and provided some useful insights (Ali \& Kohun, 2005; Ali \& Kohun, 2006; Ali \& Kohun, 2007; Mishra, Cellante, \& Kohun, 2018). This reflective paper takes a somewhat

\footnotetext{
* The author's affiliation with The MITRE Corporation is provided for identification purposes only and is not intended to convey or imply MITRE's concurrence with, or support for, the positions, opinions or viewpoints expressed by the author. Approved for Public Release; Distribution Unlimited. Case Number 19-2025. C2019 The MITRE Corporation. ALL RIGHTS RESERVED.
} 


\section{Issues in Information Systems \\ Volume 20, Issue 2, pp. 128-139, 2019}

different approach: The four co-authors are all recent graduates from this program, part of a cohort of 17 classmates that started the journey in August 2016, successfully defended their dissertations in April 2019, and graduated in May 2019 with a Doctor of Science in Information Systems and Communications degree. The goal of this paper is twofold: To share the co-authors' lived experiences as doctoral students while also presenting those experiences within the context of the Yin \& Yang Research Model. This model is reflected not only in the courses offered in this program, but also in the balanced approach the DISC program takes with respect to humanity and technology.

Each of the four authors came from different cultural, educational, and professional backgrounds, and their research interests and research study methodologies also differed. They each came from four different countries of origin and national culture (U.S., China, Malaysia, and South Korea), and their dissertations were based upon four different research methodologies: Mixed-Method, Design Science, Quantitative Method, and Qualitative Method. Despite such differences, over the long and arduous three-year journey the co-authors formed a close bond and supported one other both at on-campus residency sessions (by sharing food, drinks, jokes, ideas and concerns) and off-campus (via daily social media phone app communications in between residencies). In this paper the authors present their individual reflections, followed by the description of a Yin \& Yang Research Model and its relevance for understanding the design, structure, and success rate of both the DISC program and its graduates.

Since this paper is reflective in nature, the authors focus on sharing their personal experiences, with an admitted bias toward its positive aspects, rather than providing a rigorous, systematic evaluation of all aspects of the program; again, the purpose of this article was to evaluate the reasons for the DISC program's success over the course of two decades. Even though the authors observed first-hand, and heard from other students, some of the shortcomings and issues in this program, they regarded those as normal and natural phenomena which can reasonably be expected in any such program at any institution. Applying the Shannon-Weaver Information Theory, the authors filtered out noise from voice (Shannon \& Weaver, 1949), focusing on coursework and dissertations and "tuning out" unhelpful distractions. In keeping with the adaptive design philosophy of the program's creators, the current program director and faculty acknowledge that the program has opportunities to improve and have demonstrated a willingness to adjust and modify the program in response to changing technology and the needs of diverse student demographics.

\section{LITERATURE REVIEW}

A doctorate is a terminal academic degree that can be attained for either academic or non-academic professional purposes. Not everyone who earns a doctorate does so to teach at the college or university level; Gill \& Hoppe (2009) use the term business professional doctorate to describe a terminal degree that "serves as a qualifying or useful degree for the purpose of non-academic employment" (p. 29). In Europe, more so than in the United States, professional people in science, engineering, and high-level management jobs pursue doctorates for professional, rather than academic, purposes. "In Europe the distinction between professional and academic business doctorates is very much blurred" (Gill \& Hoppe, 2009, p. 36). The expectation that working professionals in certain nonacademic fields like engineering, senior management, or business acquire a doctorate is already quite high in such countries as Germany, an expectation likely to increase throughout the rest of the European Union as a consequence of educational standardization and other reforms stemming from the 1999 Bologna Process (Gill \& Hoppe, 2009). In contrast, in such countries as Germany, France, the Netherlands, Gill \& Hoppe (2009) found, "the business professional doctorate is virtually unknown at research-focused universities" in the United States, Case Western's Weatherhead School being the sole exception they found at that time (p. 45). One factor that may account for the desirability of business professionals possessing a doctoral degree within a particular country may be cultural. In characterizing national cultures on the Uncertainty Avoiding - Uncertainty Accepting scale, Hofstede, Hofstede, \& Minkov (2010), describe how individuals in a high Uncertainty Avoiding culture like Germany prefer specialists over generalists; having a doctorate certainly attests to the degree holder's specialized knowledge. By contrast, in a country like the U.S., which as a more Uncertainty Accepting culture falls out toward the opposite extreme of Hofstede's Uncertainty Avoiding index, there is greater acceptance of generalists and less perceived need to turn to specialists: Among business professionals, titles and degrees tend to carry less weight in the U.S. than they do in Germany.

In the United States, the attainability of a doctorate by established professionals (who may or may not have professorial career aspirations) has benefited from the introduction of executive-style doctoral programs in which 


\section{Issues in Information Systems \\ Volume 20, Issue 2, pp. 128-139, 2019}

residency requirements are structured to accommodate the lifestyle of professionals who have full-time jobs, and by the increasing availability and popularity of online doctoral programs. Gill \& Hoppe (2009) note that in addition to there being different types of doctoral programs (MD, JD, Ph.D., Ed.D., DBA, et cetera), there can be significant differences between those programs in terms of course structure, focus, duration, admission requirements, residency requirements, publishing expectations, and content delivery methodology. Although these various doctoral programs may differ from one another considerably in terms of structure, duration, content, and whether classes are conducted face-to-face or online, one statistic that has been consistent across both time and programs has been a roughly $50 \%$ attrition rate: that is, only one out of every two individuals who are accepted and enrolled in a doctoral program ever actually earns a doctorate (Mishra, Cellante, \& Kohun, 2018).

Studies of the reasons for this high attrition rate have identified such contributing factors as the students themselves, demographics of the student populations, doctoral program design and structure, rural vs. urban program locations, and financial aid availability (Ali \& Kohun, 2006; Ali \& Kohun, 2007). Another significant contributing factor Ali \& Kohun $(2006,2007)$ identified was a sense of isolation among graduate students, which they asserted is a significant, but under-studied, factor contributing to the high attrition rate in doctoral programs. They asserted that isolation was not only under-studied, it was not even a planning consideration for most doctoral program designers and managers, despite the fact that "one out of every two students who start a doctoral program do not finish it" (Ali $\&$ Kohun, 2006, p. 23). They describe the threat of isolation as occurring, for different reasons, during four distinct phases of the doctoral journey: Preadmission to enrollment (Stage 1), the first year of the program (Stage 2), the second year through doctoral candidacy (Stage 3), and the dissertation phase (Stage 4). Ali \& Kohun $(2006,2007)$ note that since the factors contributing to student during these stages may differ, doctoral program managers can help doctoral candidates effectively mitigate those factors through techniques specifically designed to target those risk factors in each stage of the doctoral journey.

Mishra, Cellante, \& Kohun (2018) note that although there are a number of universities in the U.S. alone that offer doctoral degree programs in information systems and communication, the rapidity of changes in the computer and information systems fields means that doctoral programs in those areas are constantly changing, and "no two programs are alike" (p. 170). The authors examine one particular doctoral program in information systems and communications that has maintained a better than $90 \%$ graduation rate since it was founded in 1999 , a success rate that stands in stark contrast to the roughly $50 \%$ attrition rate of all doctoral programs. That program is the Doctor of Information Science in Information Systems and Communications (DISC) program, which was established at Robert Morris University in 1999 and graduated its first students in 2002 (Kohun \& Ali, 2005). Although the residencybased RMU DISC program is what Gill \& Hoppe (2009) would characterize as a business professional degree, some RMU DISC graduates subsequently pursued successful careers in academia, while others achieved success in various non-academic, but professional, capacities.

A number of factors have been identified as contributing to the comparatively high graduation rate of the RMU DISC program. These include the program's structure, mandatory residencies, cohort enrollment approach, effective communication, and faculty engagement (Mishra, Cellante, \& Kohun, 2018). Survey responses from DISC program alumni identified the cohort format, faculty support, program structure, and flexibility in pursuing ideas as four particular strengths of the program (Mishra, Cellante, \& Kohun, 2018). The value of a cohort approach was identified by Burnett (1999), who described how the use of a cohort model contributes to communication and collaboration between doctoral cohort members, which aids them in combatting stress and a sense of isolation. The benefit of face-to-face interaction between doctoral students was also identified by Gill \& Hoppe (2009), who noted the importance of effectively socializing incoming doctoral students for ensuring "career success and avoiding attrition" (p. 51). The purpose of such socialization "is to ensure the candidate develops behaviors, values, and norms appropriate to academic life" (p. 51). In examining the role of faculty members in contributing to doctoral students' success, Samuel \& Kohun (2010) described how socialization is not only horizonal (between doctoral students), but also vertical (between doctoral students and faculty).

Although a residency-based doctoral program brings together professionally-experienced, intelligent, and highlymotivated students as a cohort focused on achieving a shared goal, individual doctoral students' personalities, cognitive styles, and coping behaviors still play an important role in shaping their experience, and ultimate success, in the program (Samuel \& Kohun, 2010). Although an experienced professional entering a doctoral program is already a professional-expert, the intellectual demands of a doctoral program push all students into a discomfort 


\section{Issues in Information Systems \\ Volume 20, Issue 2, pp. 128-139, 2019}

zone (Samuel \& Kohun, 2010). Another important role played by the socialization process not explicitly identified by Gill \& Hoppe (2009), but especially valued in a residency program, is the bonding of cohort members achieved through face-to-face communication and shared experiences over the multi-year program, a period of time in which individual students not only acquire knowledge but also undergo psychological transformations as well. Indeed, part of the transition that cohort members undergo during the three years of the program is a mental transition "from a job perspective to a career perspective" (Samuel \& Kohun, 2010, p. 35).

Capturing the observations, thoughts, and insights of individuals who have recently undergone certain experiences is common in many organizations, and has long been standard operating procedure in the U.S. military, where service members who have very recently completed an operation, deployment, military operation, or training course are asked to share and reflect upon their experiences and observations. For example, it is standard practice for U.S. Army Special Forces soldiers returning from overseas deployments to undergo debriefing under the Special Operations Debrief and Retrieval System, or SODARS (ATP 3-05.2, 2015). Additionally, the U.S. Army also has a Center for Army Lessons Learned at its Combined Arms Center at Fort Leavenworth, Kansas (http://usacac.army.mil/cac2/call/index.asp), which not only serves as a repository for accumulated After Action Reports (AAR), but also as a centralized venue where experienced Army personnel access AARs and other historical documents to assess tactics, techniques, and procedures for the purpose of identifying what can be improved, and done better in the future.

\section{REFLECTIONS OF AUTHOR ONE}

The students comprising Cohort 18 came from a broad spectrum of professional and academic backgrounds and possessed varied technical aptitude and experience in such IT-related skills as programming, database management, systems engineering, information security, et cetera. Fortunately, one of the recognized strengths of this program was the fact that it could successfully accommodate, and benefit, students with such varied backgrounds, skills, and experience. For example, although I had an adequate, high-level understanding of information technology, I did not have any of the specialized IT skills (such as programming, database management, or cyber security) possessed by many of the cohort colleagues. Conversely, though, I did have relevant experience in some other areas that my colleagues lacked. I found the diversity of our cohort members' backgrounds helped to enrich the learning experience of all cohort members, who in class discussions often learned as much from experienced colleagues as from the professor teaching the class. However, this strength could also be perceived by some as a weakness if an incoming doctoral student was anticipating a highly-technical program of study in a narrowly-focused IT field: A narrowly-focused, technical education was not the intent of the program's designers.

Also contributing to the success of both the program and its individual doctoral students has been the fact that the majority of cohort members were already well-established and experienced in their respective professions, and entered the program with at least some general idea as to what the subject of their research study would be ("information security", "autonomous vehicles", et cetera). Because of the program's structure, students generally knew by the end of the first year what they wanted to study; by the end of the second year, they also knew how, because by that point it was necessary to select, justify, and defend their selected research design. In contrast to some doctoral programs, students in this particular program have broad latitude in selecting the research topic for the fieldwork that forms the basis for the required doctoral dissertation.

Several of the professors who originally founded the program two decades ago continue to play active roles as course instructors, dissertation advisors, or committee members, and by providing input regarding the sequencing and content of individual classes. The stability and continuity provided by those "plank holder" professors, coupled with fresh perspectives and experiences provided by more recent additions to the staff, help to keep the program quality consistent and individual course content relevant. In my case, the continued, active involvement of the program's founders proved essential for the success of my research study: Performing a mixed-methods research study involving two separate sample populations on two different continents would be a challenging undertaking under any circumstances; attempting this during the last half of a three-year-long, executive doctoral program (i.e., on top of full-time employment and family commitments) proved to be especially challenging, especially after a number of well-known multinational firms declined to participate in a proposed study that would identify and assess the information security attitudes of their employees. Fortunately, the global academic networks and personal 


\section{Issues in Information Systems}

Volume 20, Issue 2, pp. 128-139, 2019

relationships developed over time by DISC program founders/professors made it possible to identify and secure the cooperation of suitable corporate study participants and thereby ensure the timely completion of my research study and the 340-page dissertation documenting it.

In addition to support from the faculty, students in this program benefited in several ways from peer engagement. The cohort approach, coupled with the face-to-face interaction made possible by the residency interactions, provided cohort members and opportunities to communicate, collaborate, exchange information and ideas, and sometimes vent. We made extensive and regular use of the social media platform WhatsApp to stay in touch throughout the three years of the program.

One particular aspect of this program which I found indispensable was its peer mentoring component, which as Mishra, Cellante, \& Kohun (2018) assert is intended to "provide a safe environment for giving and receiving feedback" (p. 177). During the first semester of the first year, all cohort members were instructed to select a fellow cohort member to serve as a peer mentor. In my case, I was extremely fortunate to have selected a peer mentor whose strengths, experience, and knowledge were in areas where I was academically or experientially deficient; in several of the courses, my peer mentor and I met outside of residencies to review course materials and jointly complete assignments which I would have never been able to accomplish on my own. The peer relationship functioned so well that we co-authored multiple articles that were published in peer-reviewed scholarly journals even before we graduated from the doctoral program.

As Ali \& Kohun $(2006,2007)$ observed, doctoral candidates are not only older and forced to balance their academic lives with significant familial and professional responsibilities, they also must often contend as well with a sense of isolation. At two different (and critical) points in my own doctoral journey, I unexpectedly learned I was about to lose my job. Fortunately, the emotional support provided by fellow cohort members and faculty in both cases proved indispensable in helping me not only combat fear and isolation, but also remain on track and continue to perform my academic work, both individually and on team projects. When others in the cohort similarly encountered professional, academic, or familial challenges during the course of the program, I joined with fellow cohort members to provide emotional support and lend a hand, however possible. The bond our cohort members established early on in the program was comparable to that which I was fortunate enough to experience as a member of several elite military units; this bond and emotional support enabled my cohort peers and me to benefit from the cohort experience in ways described by Burnett (1999).

\section{REFLECTIONS OF AUTHOR TWO}

Prior to joining the program, my educational background had been mostly in Science-Technology-EngineeringMathematics (STEM) and my professional experience had been primarily in information technology as a software engineer, systems engineer, technical lead, and project manager. The first weeklong residency of this program is the most challenging and tiring one. The three courses (Ethnography of Information Systems, Information Age Organization, and Rhetorical Analysis) have less to do with technology and more to do with philosophy, sociology, and humanity. This really knocked me out of my comfort zone. The Ethnography of Information System is the most challenging one. Like most people in the technical field, this is the first time I ever heard of the word "ethnography", let alone understood what it is. The course covered anthropology, field study, symbolism, and different worldviews including positivism, interpretivism, social constructivism, and pragmatism. I was a bit lost and started asking about what the nature of the doctoral program is. I came with an expectation of an engineering or technology-oriented program, but it seemed to me it was more of a social science program. The value of the ethnography course started to manifest itself when we took the User-Centered Design (UCD) and Usability Testing course in the second semester. We learned that ethnography is an important foundation for understanding user experience and performing usability tests. UCD is more about humanity than technology, and "puts human needs, capacities, and behavior first, then designs to accommodate those needs, capacities, and way of behaving" (Norman, 2013, p. 8).

Throughout the three-year journey, the theme of the intertwining technology and humanity played out each semester and in every course. The Knowledge Management (KM) course is centered around the distinction and conversion between tacit and explicit knowledge. Explicit knowledge is codified, described, and expressed knowledge that represents the technical aspect of KM while tacit knowledge is the intangible, implicit, and unspoken knowledge 


\section{Issues in Information Systems \\ Volume 20, Issue 2, pp. 128-139, 2019}

that is embodied in our individual personhood and represents the humanity aspect of KM (Polanyi, 1966; Nonaka \& Takeuchi, 1995). Even in technology-oriented courses like Data Warehousing and Analytics, where computer science, information technology, statistics, and machine learning provide the technical foundation, we saw the importance of humanity as the value-proposition and guiding compass to ensure the judicial and effective use of Big Data Analytics and Artificial Intelligence (AI).

The courses were not the only thing in the program designed with a dynamic balance of technology and humanity; the cohort-based, in-person, executive-style format provided the necessary collaborative and supportive environment for cross-pollination and knowledge creation: We all stayed in the same hotel, ate three meals and snacks together, took the same courses, and followed the same dissertation schedule over the three-years. We all came from different walks of life and had diverse cultural and intellectual backgrounds. I have learned from both the doctoral faculties and my cohort colleagues. I learned so much from their presentations of a wide variety of topics in the classroom during every semester. Their critiques of my class presentations only made me a better student and scholar. Their counsel and support also helped me get through the challenging journey without feeling isolated, lonely, or helpless. Each of them enriched both my life experience and my intellectual capacity.

One unique characteristic of this program is its flexibility. Students have the freedom to choose a wide variety of dissertation topics and choose any research method that they have passion for and feel best fit their skill set and time constraints. The faculties including the advisors and committee members encouraged us to find our passion and pursue what is meaningful for us. They provided guidance and support during our dissertation writing process. I am more of a pragmatist and decided to choose Design Science Research (DSR). Even though DSR is not prevalent in a social/behavioral science program, my advisor and committee members did not persuade me to change to the traditional qualitative or quantitative method. The good news is that DSR and behavioral science research are complementary. I was able to incorporate expert review which is a qualitative method as an evaluation method for my design of a data analytics process model.

\section{REFLECTIONS OF AUTHOR THREE}

Looking back on the very first day of residency, I was amazed by the different ethnic backgrounds of the composition of the cohort. Not only were the cohort members varied physically and experientially, we were also very diverse in our thinking and approaches to life. This diversity adds to the value of our collective contributions towards the DISC program. The very first class on "Information Age Organization" provided us with foundational information system, organizational and management theories. Here we were exposed to the first, second, and "N" numbers of consequences, the interconnectedness and multi-dimensional knowledge base, as well as the paradoxes of technology. This course set the tone for the three-year doctoral program and changed our ability to think conceptually and abstractly down to the applicable levels. Together with the understanding and application of the Shannon-Weaver model of communication, we were able to achieve a higher level of learning. Prior to obtaining the doctoral degree, I thought that I had learned all that I needed by earning a Master of Science degree in Information Systems Technology. I was dead wrong. The knowledge and the ability to think beyond human capability is something that we should all strive for - and this doctoral program does enable all of us to do so.

The course diversity gives students the ability to think more holistically than would be possible via a siloed approach. A sampling of the doctoral course curriculum includes subjects that utilize our right brain - "Science and Myth", "Knowledge Management", and "Ethnography", and subjects that cover the practical use of our left brain "Economics of Information System", "Field Project Research", and "Research Methodology." Collectively, the range of subjects and the balance of "technical" versus "non-technical" subjects enhance the value of the doctoral education. To survive and surpass our human's capabilities, one needs to broaden the horizon and widen the knowledge in order to effectively mitigate and minimize life's challenges. In the worlds of technology advancements, problems need to tackle from "people-process-technology" perspective, as opposed to just a single aspect of this Golden Triangle of business considerations.

Growing up during my early childhood in South East Asia, and having spent most of my adulthood in the United States, has contributed to my ease of exercising both my left and right brain. With that, my choice of selecting a research methodology as part of my doctoral dissertation is one that is not of a preference, but of practicality. The used of a quantitative method in my dissertation was based upon my research topic and questions, and the method 


\section{Issues in Information Systems}

Volume 20, Issue 2, pp. 128-139, 2019

employed in collecting the needed data. The choice of a quantitative research methodology fits well with my study, especially with the sampled population, which is geographically diverse.

The sequence in which the individual DISC program courses were taught has a purpose: The courses are arranged in a set order to ensure that students obtain the necessary knowledge to proceed to the next step of writing the doctoral dissertation. Like each building block for building a Lego toy, each subject provides the incremental knowledge to achieve the final outcome of a doctoral dissertation. The assignment of a dedicated one-to-one advisor at the early part of the doctoral journey helps guide the student to avoid pitfalls and to overcome challenges in conducting the research. All the advisors and professors are available at all times to answers any questions that the students may have. The quality of professors is astounding - most of them are tenured professors, some with over 30 plus years of teaching, are well-published in established journals, with some recognized as seminal figures in their individual fields of expertise. Apart from all their well-deserved accolades, the utmost important shared attribute all DISC program professors possess is a clear desire to ensure the success of all their doctoral students. It is this last attribute that I feel distinguishes them from professors in other universities, an attribute that enabled me to become a member of the "doctoral club" at the end of this three-year-long journey.

\section{REFLECTIONS OF AUTHOR FOUR}

As one of few students in Cohort 18 who was already working as a researcher in a laboratory environment involving scientific and engineering research (i.e., focused on technical engineering research), I found the first semester of the program to be extremely challenging. Unlike many students in the program, I wasn't interested in becoming a professor at a university or seeking to advance to a senior executive position. Rather, I came to the program to switch my career and research area from one field to another. As someone who has been involved with conducting and supporting Research and Development (R\&D) efforts within a federal government STEM organization that primarily relied on quantitative research methods, the courses from the first semester raised questions like "Why is this relevant to the Information Systems?" and "How come I am not being taught material on Information Systems?". With many doubts that continuously increased throughout the first semester, I questioned my decision to enroll in the program rather quickly, which was further fueled by periodic discussions with my $\mathrm{PhD}$ colleagues who questioned non-technical courses, short duration, and lack of technical value from the Doctor of Science program when compared to the traditional $\mathrm{PhD}$ programs. After contemplating a quick exit from the program several times throughout the first semester, I slowly realized that it is not about the content of the courses that mattered, but the experience I gained from other technical experts within the cohort as well as learning how to conduct research using non-quantitative methods; this was where the advisor and other professors proved to be instrumental.

My initial dissertation topic was related to drone swarms research I was already performing in the laboratory. I knew what I wanted to do even before the program started, as I planned to leverage duties from my job, which allowed me to continue obtaining financial support for the tuition cost from my organization. This plan vanished slowly as I realized in the beginning of second year that I need to think outside of my comfort zone and start learning and, more importantly, doing something new. This was further solidified when I was informed I can't publish my initial dissertation topic without restrictions. Even though I was in an immediate crisis to find another dissertation topic almost a semester behind everyone else, in hindsight, this was blessing in disguise as I was forced out of my comfort zone by finding a topic that would utilize qualitative research method that also applied to the research being done at my organization. The new topic came from one of the cohort colleagues and my advisor was instrumental in linking the topic to my duties so it may become impactful to my organization. Once again I realized that this program was about the experience and people and less about learning technical contents on Information Systems.

After successfully defending my new dissertation topic, I realized I now have a new arsenal of tools in my knowledge drawer that I can apply both in my current position as well as in any new position that may come my way. More importantly, I learned how to perform qualitative research, which provides whole new insights that can only enrich and supplement findings from technical research efforts in which my organization is currently involved, to include current research efforts on Artificial Intelligence (AI) and Machine Learning (ML). Current research efforts focus on the technical engineering and scientific aspects, but as engineering and scientific aspects of AI and ML become mature, the human aspects of how AI and ML will impact and interact with humans need to be researched, for which qualitative technique may provide additional insights. As I noted previously, this program is 
about the experience, people, and thinking out-of-box, especially for those who have been involved in a technical track throughout their careers. As I realized in the final year, if I wanted to simply become a technical expert in Information Systems, I should have pursued PhD program. While the RMU DISC program ultimately met my goal of earning a terminal degree, more importantly, it gave me whole lot more as I feel I am a much stronger researcher who now understands the qualitative aspect of research and the value of qualitative research-derived findings.

\section{THE YIN AND YANG RESEARCH MODEL}

If there is one lesson the authors learned from the DISC program that is the most important and practical, it would be the value of theories and models to examine, understand, and explain phenomena. With this in mind, the authors took the concept of Yin and Yang from the ancient Chinese culture of thousands of years and integrated it with the modern Western concept of Data-Information-Knowledge-Wisdom (DIKW) hierarchy to develop the Yin \& Yang Research Model as a framework to anchor the various doctoral courses offered in this program. The authors had no prior knowledge as to what the original founders of this program had in mind when they designed this program twenty years ago. However, by applying the Yin \& Yang Research Model, they hoped to gain some insight as to the founders' thinking and intentions. This model turned out to be very revealing and insightful; the courses that firstyear doctoral students once considered random and perhaps even irrelevant now appear to have been strategically chosen and sequenced, and their inclusion and ordering makes great sense. By reverse engineering the design of the DISC program in this manner, the authors hoped to gain improved understanding of the reasons for its success and inform other institutions and programs that may be seeking ways to design or improve their own doctoral $\operatorname{program}(\mathrm{s})$.

The ancient concept of Yin and Yang is the simplest, yet most powerful, model of all; as a foundational concept, it is on par with the role played by zero and one in computer science. Yin-Yang balance is the most fundamental concept of the ancient Chinese philosophy. Many aspects of Chinese civilization from culture to politics, from science to medicine, and the many schools of thoughts including Taoism and Confucianism have been deeply rooted in, or subtly influenced by, this concept.

Yin and Yang are the most basic elements or forces that make up or drive the phenomenal reality - physical and metaphysical. The interaction, interconnection, and interdependence of the two opposing but complementary and inseparable elements or forces is the simplest possible but most elegant way to explain phenomena of any kind, be it cosmos, nature, human, or society. Human life is the balancing act of Yin and Yang and requires the embracement of a holistic, dynamic, and dialectical duality (Li, 2008, p. 416).

While there are many variations of Yin Yang symbol, Figure 1 shows one that is simple and intriguing.

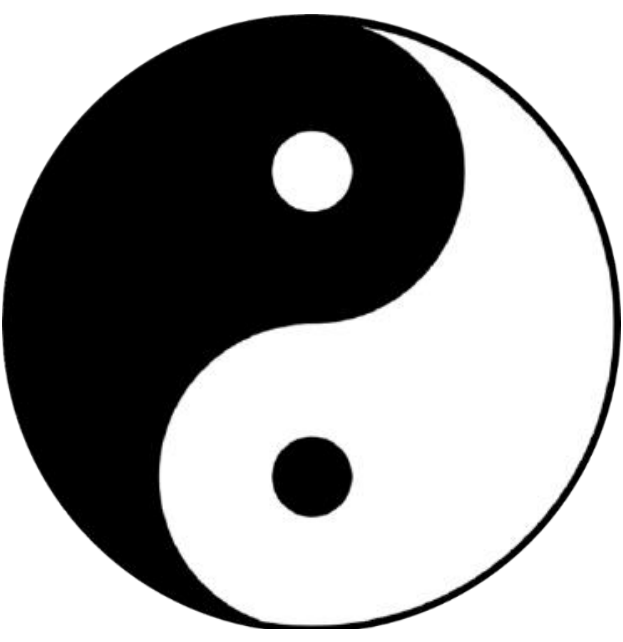

Figure 1. The Yin-Yang Symbol 


\section{Issues in Information Systems}

Volume 20, Issue 2, pp. 128-139, 2019

The imbalance of Yin and Yang is the root of instability. For the whole which both Yin and Yang are part of to survive, sustain, and prosper, Yin and Yang must constantly interact with each other and complement each other to reach the ever-evolving dynamic balance. Interestingly, the globe is divided geographically into two hemispheres the East and the West. These two hemispheres also exhibit prominent differences in cultures, religions and economic standings. The West has the characteristics of the Yang, which is masculine, individualist, extrovert, analytical, and innovative. The East has the characteristics of the Yin, which is feminine, collective, introvert, intuitive, and conservative. Consequently, the West is more advanced in science and technology, more economically developed and more active and aggressive in world affairs; In contrast, the East tends to focus more on humanity, arts, selfcultivation, harmony between human and nature, harmony within self, family and society.

The authors applied the ancient concept of Yin and Yang along with the well-known modern Data-InformationKnowledge-Wisdom (DIKW) hierarchy (Zeleny,1987; Ackoff, 1989) to academic study and research and developed the Yin Yang Model of Research as depicted in Figure 2.

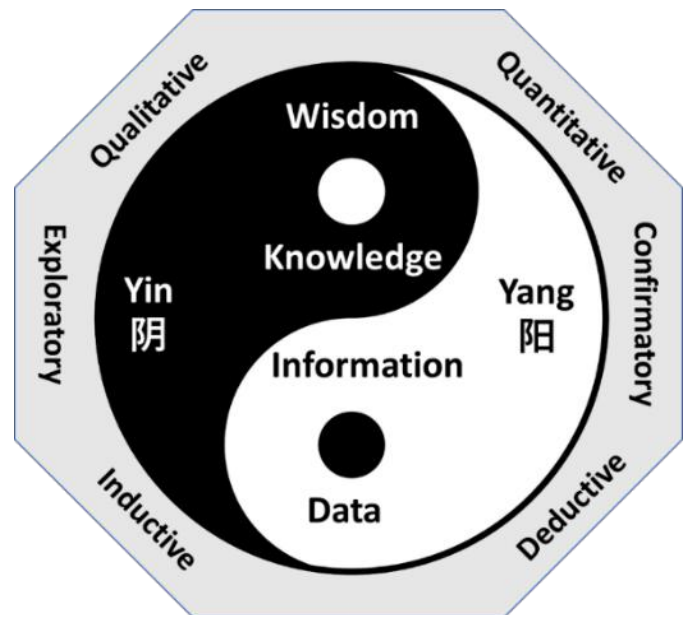

Figure 2. The Yin and Yang Model of Research

In the Yin and Yang Model of Research, data and information are considered Yang since they represent explicit knowledge that can be explicated, expressed and codified. Knowledge and wisdom represent tacit knowledge that humans embody and cannot be explicitly expressed and codified. Quantitative research methodologies are confirmatory and deductive in nature and start with a theory, model or hypothesis and seek evidence from data for confirmation through falsification of the null hypothesis. Quantitative approach is placed on the Yang side. On the other hand, qualitative research methodologies are exploratory and inductive in nature and seek to discover and derive knowledge and theory from data and information. The qualitative approach is placed on the Yin side. Yin and Yang are not mutually exclusive, they are two sides of a coin and co-exist and complement each other. There is Yin in Yang as indicated by the small black dot and Yang in Yin as indicated in the small white dot in the figure. Mixed methodology reflects this reality. In general, all research endeavors regardless of methods used incorporate both qualitative and quantitative elements and the differences are the degree and depth of the elements.

Figure 3 shows the Yin Yang Model of Research as being used to anchor all doctoral courses offered by the program. 


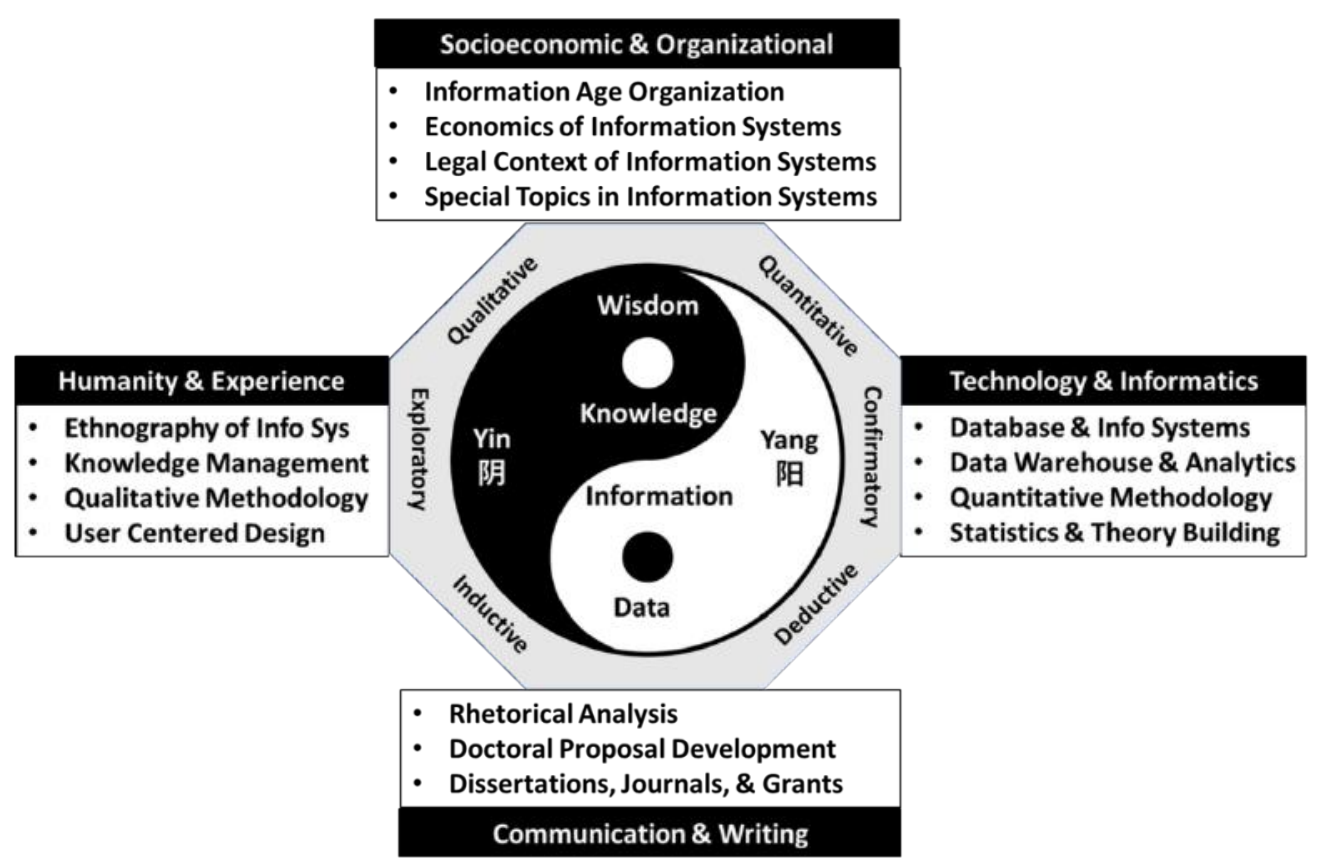

Figure 3. The Yin and Yang of the DISC Program at RMU

The doctoral courses are categorized in four dimensions:

- Humanity \& Experience: These courses focus on human factors, human experiences and hence is placed on the Yin side.

- Technology \& Informatics: These courses focus on statistics, information technology, data management and analytics. They are placed on the side of Yang.

- Socioeconomic \& Organizational: These courses integrate technology with humanity and place technology in the context of socioeconomic and organizational context. They are placed at the top closer to wisdom in the DIKW hierarchy.

- Communication \& Writing: These courses provide students with theoretical foundation for effective communication, scholarly writing, and process of proposal development for dissertations.

As seen in Figure 3, these courses are distributed across the spectrum between Yin (humanity and experience) and Yan (technology and informatics).

On the left-side, the courses about Humanity \& Experience (Yin) are:

- Ethnography of Information Systems

- Knowledge Management

- Qualitative Methodology

- User-Centered Design

On the right-side, the courses about Technology \& Informatics (Yang) are:

- Database and Information Systems

- Data Warehouse and Analytics

- Quantitative Methodology

- Statistics \& Theory Building

At the top of the Yin-Yang Research Model, the courses align with Knowledge and Wisdom of the DIKW hierarchy are focused on Socioeconomic and Organizational context of information technology and systems:

- Information Age Organization

- Economics of Information Systems 
- Legal context of Information Systems

- Special Topics in Information Systems

At the bottom of the Yin-Yang Research Model, the courses align with Data and Information of the DIKW hierarchy and are focused on Communication \& Writing:

- Rhetorical Analysis

- Proposal Development

- Dissertations, Journals, \& Grants

Based upon the courses positioned in Figure 3, one can see that the courses offered by this program are well balanced - covering both technology and humanity. In addition, the techniques of communication and writing are also complemented with the coverage of socioeconomic and organizational context. Overall, the design of the program is well-balanced following the harmonious nature of Yin and Yang. It is worth noting that the gender of the professors teaching the courses on each of the four dimensions is evenly distributed among male and female professors. The authors were very much amazed by this serendipitous discovery.

\section{CONCLUSION}

While traditional doctoral programs chronically suffer from high attrition rates, the Doctor of Science in Information Systems and Communications at Robert Morris University has consistently enjoyed a graduation rate of over $90 \%$. Factors contributing to this high graduation rate include the cohort-based, residency format that provides a collaborative and supportive environment for experienced and accomplished working professionals. While several papers have been written about the program from various perspectives and using different methodologies, this paper is unique in that it is written by four recent graduates and provides fresh accounts of their personal experiences and reflections. In addition, this paper presents a Yin \& Yang Research Model integrating the ancient Chinese concept of Yin and Yang with the modern Western concept of Data-Information-Knowledge-Wisdom (DIKW) hierarchy. This paper categorized the doctoral courses offered by the program into four dimensions and anchored them along this model. This helped to reveal interesting patterns about the program design of the balanced distribution of courses along humanity and technology. While four current graduates represent an admittedly small and biased sample of over two hundred graduates, the authors believe their reflections, considered within the framework of the Yin \& Yang Research Model, provide valuable insights useful to better understand some of the reasons for the program's consistently high graduation rate. Such insights may be useful to other institutions seeking to establish a new, similar program or to improve an existing one. In addition, this paper provides practical and theoretical perspectives to help prospective students in their pursuit of a terminal degree, whether they are still in the process of program selection or already undertaking the journey of higher learning.

\section{REFERENCES}

Ali, A., \& Kohun, F. (2006). Dealing with isolation feelings in IS doctoral programs. International Journal of Doctoral Studies, 1, 21-33.

Ali, A., \& Kohun, F. (2007). Dealing with social isolation to minimize doctoral attrition: A four stage framework. International Journal of Doctoral Studies, 2, 33-49.

ATP 3-05.2, Foreign Internal Defense (2015, August). Washington, DC: Department of the Army. URL: https://fas.org/irp/doddir/army/atp3-05-2.pdf, accessed 7 May 2019.

Burnett, P. C. (1999). The supervision of doctoral dissertation using a collaborative cohort model. Counselor Education and Supervision, 39(1), 46-52.

Gill, T. G., \& Hoppe, U. (2009). The business professional doctorate as an informing channel: A survey and analysis. International Journal of Doctoral Studies, 4, 27-57. 


\section{Issues in Information Systems}

Volume 20, Issue 2, pp. 128-139, 2019

Hofstede, G., Hofstede, G. J., \& Minkov, M. (2010). Cultures and organizations: software of the mind: intercultural cooperation and its importance for survival. New York: McGraw-Hill.

Li, P. P. (2008). Toward a geocentric framework of trust: An application to organizational trust. Management and Organization Review, 4(3), 413-439.

Kohun, F., \& Ali, A. (2005). A doctorate degree program in information systems of a kind. Issues in Informing Science \& Information Technology, 2, 179-188.

Mishra, S., Cellante, D., \& Kohun, F. (2018). Thriving in the landscape of doctoral programs: The case of D.Sc. at Xuniversity. Issues in Information Systems 19(1), 170-179.

Nonaka, I., \& Takeuchi, H. (1995). The knowledge-creating company: How Japanese companies create the dynamics of innovation: Oxford University Press.

Polanyi, M. (1966). The tacit dimension. London: The University of Chicago Press.

Shannon, C., \& Weaver, W. (1949). The mathematical theory of communication. Urbana, IL: University of Illinois Press. 